\title{
Formation of one-dimensional molecular chains on a solid surface: Pyrazine/Si(001)
}

\author{
M. Shimomura,* D. Ichikawa, and Y. Fukuda \\ Research Institute of Electronics, Shizuoka University, 3-5-1 Johoku, Hamamatsu 432-8011, Japan \\ T. Abukawa, T. Aoyama, and S. Kono \\ Institute of Multidisciplinary Research for Advanced Materials, Tohoku University, 2-1-1 Katahira, Aoba-ku, Sendai 980-8577, Japan
}

(Received 14 March 2005; published 6 July 2005)

\begin{abstract}
We have studied the chemisorption of pyrazine on $\mathrm{Si}(001)-2 \times 1$ at room temperature using scanning tunneling microscopy (STM), photoelectron diffraction (PED), and the density functional theory (DFT). In STM, protrusions ascribed to the pyrazine adsorbate were observed between the Si dimer rows and arrange themselves one-dimensionally (1D) along the dimer bond direction. Multiple scattering analysis of N $1 s$ and $\mathrm{C} 1 s$ PED patterns showed that a double-dimer bridging (DDB) configuration, in which pyrazine molecule forms bridging between the dimer rows with two $\mathrm{Si}-\mathrm{N}$ bonds, is formed. DFT cluster calculations showed, however, that $\mathrm{N}$-end-on configuration is more stable than the DDB configuration for a single molecule adsorption. The stability for the DDB configuration as a 1D chain is discussed on the basis of "polymerization" of the adsorbates through dangling bonds at the distal points of the 1D chain.
\end{abstract}

DOI: 10.1103/PhysRevB.72.033303

PACS number(s): 68.43.Bc, 61.14.Qp, 68.37.Ef

It is well known that a clean $\mathrm{Si}(001)-2 \times 1$ surface exhibits a tilted-dimer structure that accompanies electronic charge transfer from a depressed dimer atom, called down atom, to a protruding dimer atom, called up atom. ${ }^{1,2}$ It is also accepted that the tilted dimer oscillates at room temperature (RT). ${ }^{3}$ Numerous studies on reactions of organic molecules with the Si dimer have been performed to establish an association between biochemistry and silicon nanotechnology. Recently, such studies were systematically reviewed by Filler and Bent. ${ }^{4}$ Adsorption of aromatic compounds on Si has attracted attention for not only a prototypical reaction of a $\pi$-conjugated system with the dimer but also the formation of highly ordered conducting polymers on the semiconductor. Two chemisorption structures were reported for benzene $\left(\mathrm{C}_{6} \mathrm{H}_{6}\right)$ on $\mathrm{Si}(001)-2 \times 1$. It was reported that a di- $\sigma$-bonded structure was formed by the $[4+2]$ cycloaddition reaction at RT (Refs. 5-8) and that the di- $\sigma$ configuration falls into a tetra- $\sigma$ configuration. ${ }^{9-11}$ A few studies have been reported on the adsorption of nitrogen-containing aromatic molecules on $\mathrm{Si}(001)-2 \times 1 .{ }^{12-14}$ For pyridine $\left(\mathrm{C}_{5} \mathrm{H}_{5} \mathrm{~N}\right)$, which contains one nitrogen atom in the six-membered ring, an $\mathrm{N}$-end-on (NEO) and a di- $\sigma$ configurations are found at the local energy minima using the density functional theory (DFT). ${ }^{12,13}$ In the NEO configuration, the $\mathrm{N}$ end of the pyridine is datively bonded to the down atom of the dimer. Theoretical ${ }^{12}$ and experimental ${ }^{14}$ studies have recently been reported for pyrazine $\left(\mathrm{C}_{4} \mathrm{H}_{4} \mathrm{~N}_{2}\right)$, which contains two nitrogen atoms located at the para position. The theoretical investigation using DFT with a $\mathrm{Si}_{9} \mathrm{H}_{12}$ cluster ${ }^{12}$ suggests that the NEO configuration (Fig. 1, N-end-on) can be considered as the primary adspecies at low temperature while the main configuration can be changed to the 2,5-di- $\sigma$ configuration (Fig. 1, 2,5-di$\sigma)$ at elevated temperatures. On the other hand, highresolution electron energy loss spectroscopy (HREELS) and $\mathrm{X}$-ray photoelectron spectroscopy (XPS) study $^{14}$ suggested recently that the chemisorption occurs through the bonding of two nitrogen atoms with a dimer. Thus, the 1,4-di- $\sigma$ con- figuration (Fig. 1, 1,4-di- $\sigma$ ) is experimentally supported.

In the present work, we have investigated the surface chemistry of pyrazine on $\mathrm{Si}(001)-2 \times 1$ using scanning tunneling microscopy (STM), photoelectron diffraction (PED), and the first principle DFT calculation and a definite model of the chemisorption is found. Namely, the double-dimer bridging (DDB) configuration (Fig. 1, Double-dimer bridging) is the correct model and the chemisorbed species line up one-dimensionally (1D) along the dimer bond direction. We further discuss the formation mechanism of 1D molecular chain.

The STM and PED measurements were performed using different apparatuses: An STM apparatus (JEOL, JSTM4500XT) equipped with chemically etched W-tip and a PED spectrometer (VG-CLAM4) installed on the beam-line 13C of the synchrotron radiation facility KEK-PF, Tsukuba, Japan. The PED spectrometer was equipped with a five-axis sample manipulator, angular rotations of which were controlled by a computer for PED measurements. The STM and PED measurements were carried out under an ultrahigh vacuum (UHV) condition $\left(<2 \times 10^{-10}\right.$ Torr $)$ at RT. The Si samples were cleaned by flash heating up to $\sim 1250{ }^{\circ} \mathrm{C}$. In

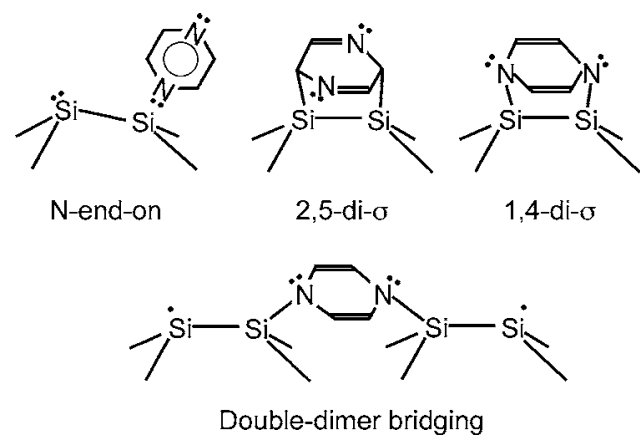

FIG. 1. Scheme of chemisorption models of pyrazine on $\mathrm{Si}(001)-2 \times 1$. Lone pairs and unpaired electrons are represented by double and single $\operatorname{dot}(\mathrm{s})$, respectively. 
the PED measurement, the sample was postannealed at $\sim 1000{ }^{\circ} \mathrm{C}$ to obtain a single-domain (SD) $2 \times 1$ surface. Low-energy electron diffraction (LEED) spot intensities verified that the major domain percentage was $\sim 90 \%$. This percentage was considered in all the PED simulations. The clean $\mathrm{Si}(001)-2 \times 1$ surface was exposed to pyrazine gas at RT for the STM and PED experiments. The PED experiment was performed at the saturation coverage. Angle-scanned PED patterns were compared with the results from multiple scattering simulation for model clusters. The Multiple Scattering Calculation of Diffraction (MSCD) package ${ }^{15}$ was used for the PED simulation. Hydrogen atoms were neglected in the simulation since they only negligibly affect the scattering. In both the PED experiment and simulation, the anisotropy function was assumed as the following equation for the polar $(\theta)$ and azimuthal $(\phi)$ angles: $\chi(\theta, \phi)$ $=\left[I(\theta, \phi)-I_{0}(\theta)\right] / I_{0}(\theta)$, where $I(\theta, \phi)$ and $I_{0}(\theta)$ denote the photoelectron intensity and the mean intensity for a given polar angle, respectively. A gradation image was obtained by plotting the anisotropy functions of the experiment and simulation. The analysis of the experimental data was performed with an $R$ factor defined as

$$
R=\frac{\sum_{i}\left(\chi_{\text {sim }}-\chi_{\text {expt }}\right)^{2}}{\sum_{i}\left(\chi_{\text {sim }}^{2}+\chi_{\text {expt }}^{2}\right)}
$$

where $\chi_{\text {expt }}$ and $\chi_{\text {sim }}$ are the normalized anisotropy functions of the experiment and simulation, respectively. The chemisorption energies of the models were calculated by the DFT using the GAUSSIAN 03 package ${ }^{16}$ with hybrid functionals consisting of Becke's three-parameter nonlocal exchange functional and the Lee-Yang-Parr correlation functional (B3LYP). ${ }^{17,18}$ The standard 6-31G(d) basis set ${ }^{19,20}$ was used in the calculation.

Figures 2(a)-2(c) show the STM images obtained with an exposure of $0.3 \mathrm{~L}\left(1 \mathrm{~L}=1 \times 10^{-6} \mathrm{Torr} \cdot \mathrm{s}\right)$. Elliptical protrusions are sparsely observed in the large-scale STM image [Fig. 2(a)]. The number of protrusions increases with the exposure. Thus, it is highly probable that these features correspond to pyrazine adsorbates. Figure 2(d) shows a schematic representation of the magnified image in Fig. 2(b). In Fig. 2(d), "A," "B," and "C" denote the protrusion of the pyrazine adsorbate, vacancies of Si dimers, and static buckling of dimers caused by defects, ${ }^{21}$ respectively. In Figs. 2(b) and 2(d), dashed lines are inserted directly above the $\mathrm{Si}$ dimer bonds, estimated by the positions of " $\mathrm{B}$ " and " $\mathrm{C}$;" solid lines are also inserted above the center of the $\mathrm{Si}$ dimer rows. It was found that the protrusion center is located at the halfway between the dimer rows along the line connecting the dimer bonds. Over $90 \%$ of the protrusions were located at the same site. At saturation coverage, almost all the pyrazine species were also found to be at the same site (data not provided here). Consequently, chemisorption models in which the pyrazine species is located on a Si dimer or two adjacent dimers in the same row, such as di- $\sigma$ or tetra- $\sigma$ configurations, can be excluded as the major structure.

Thus, the relative position of the adsorbate to the dimers was clarified by the STM results. Information about orientation of each adsorbate is required for proposing a new model.
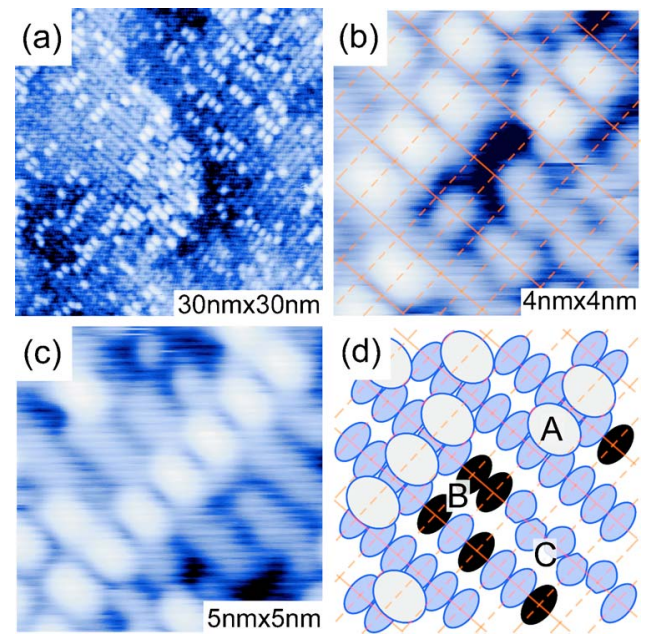

FIG. 2. (Color online) STM images of the pyrazine-adsorbed $\mathrm{Si}(001)-2 \times 1$ surface at an exposure of $0.3 \mathrm{~L} . V_{\text {sample }}=-1.4 \mathrm{~V}, I$ $=0.3 \mathrm{nA}$. (a) Large-scale image. Atomic-scale images showing (b) the pyrazine adsorbates near Si defects and (c) the 1D molecular chain. (d) Schematic presentation of (b): "A," "B," and "C" denote the protrusion of the pyrazine adsorbate, vacancies of Si dimers, and static buckling of dimers, respectively.

Analyses by diffraction methods are suitable for such purposes. PED, in particular, is advantageous from the viewpoint of element isolable and local structural analyses. Here, we propose the DDB model in which pyrazine molecule forms bridging between the dimer rows with two $\mathrm{Si}-\mathrm{N}$ bonds (Fig. 1, double-dimer bridging) as the best-fitted model in PED. Figure 3 shows the N $1 s$ and C $1 s$ PED patterns obtained at photon energies of 700 and $515 \mathrm{eV}$, and 700 and $396 \mathrm{eV}$, respectively. The right- and left-hand sides of each pattern correspond to the experimental and simulated anisotropy functions, respectively. The simulation assumes the DDB model whose structural parameters were optimized by the DFT (discussed later). As shown in Fig. 3, the features of each simulation pattern of the DDB model are in good agreement with those of the corresponding experimental patterns. The average of the $R$ factors of four patterns is 0.12 , which is comparatively small. The STM image of the NEO configuration comprising a rotation axis along the $\mathrm{N}-\mathrm{Si}$ bond may exhibit a peak between the dimer rows in STM. We calculated the PED patterns of the NEO model assuming an average of the anisotropy functions in $30^{\circ}$ steps of rotation. The average of the $R$ factors of the NEO model is 0.37 , which is relatively large. The characteristics of the DDB model also agree with the results by XPS and HREELS (Ref. 14) in that (1) only $\mathrm{N}$ atoms are bonded to $\mathrm{Si}$ and (2) N and $\mathrm{C}$ are in a singular chemical environment. Prior to the PED measurement, we measured the $\mathrm{N} 1 s$ and $\mathrm{C} 1 s$ lines with high resolution $(\sim 0.3 \mathrm{eV})$ and confirmed that each of the lines is fitted by a Voigt function.

The theoretical estimation of the stability for the new model is worth reporting. The structural optimization of the NEO and DDB models was performed by the B3LYP/6$31 \mathrm{G}(\mathrm{d})$ level calculation with a $\mathrm{Si}_{23} \mathrm{H}_{24}$ cluster. The calculation result indicates that these models can be found to be at the energy minima. The optimized structures with key bond 

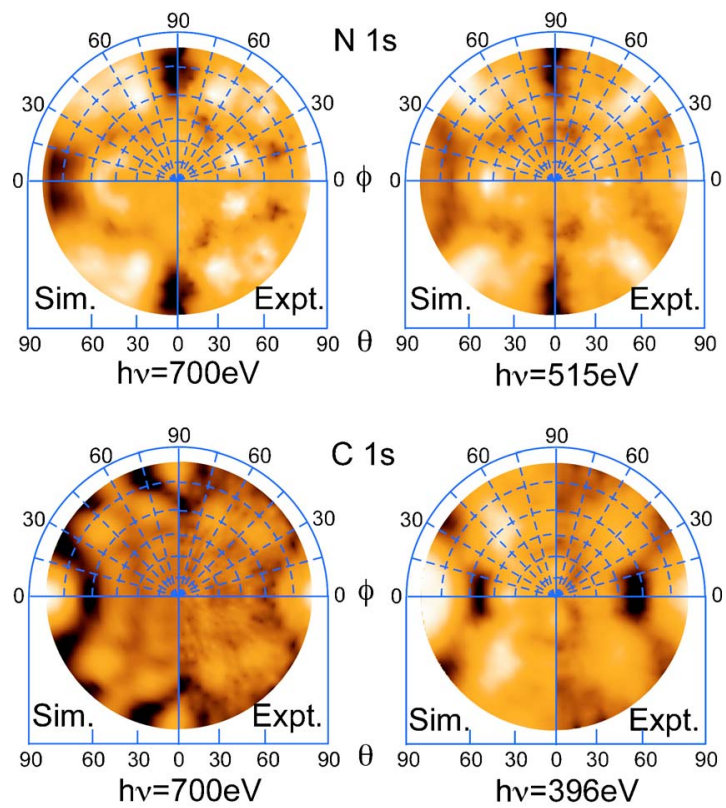

$\operatorname{Min} . \square$ Max

FIG. 3. (Color online) The N $1 s$ and C $1 s$ PED patterns of the pyrazine-saturated $\mathrm{Si}(001)-2 \times 1$ surface. Photoelectrons were collected at photon energies of 700 and 515 (396) for $\mathrm{N} 1 s$ (C 1s). In each pattern, the right- and left-hand sides correspond to the experimental and simulated anisotropy functions. The DDB model with DFT-optimized structural parameters is assumed for the simulation. The zero point of the azimuthal angle corresponds to the Si-dimer bond direction of the major $2 \times 1$ domain.

lengths and angles are shown in Fig. 4. The corresponding energy changes $(\Delta E)$ for the adsorption of pyrazine onto the cluster are calculated to be -23.1 and $-10.5 \mathrm{kcal} / \mathrm{mol}(-1.00$ and $-0.455 \mathrm{eV} /$ cluster) for the NEO and DDB models, respectively. That is, $\Delta E$ of the NEO model is smaller than that of the DDB model. It should be noted that this calculation assumes a single adsorbate. The stability of the DDB model may be strongly affected by the surrounding environment for the following reason. As shown in Fig. 1, the DDB model has a unique characteristic form in that unpaired electrons are located on those surface $\mathrm{Si}$ atoms that are not bonded to

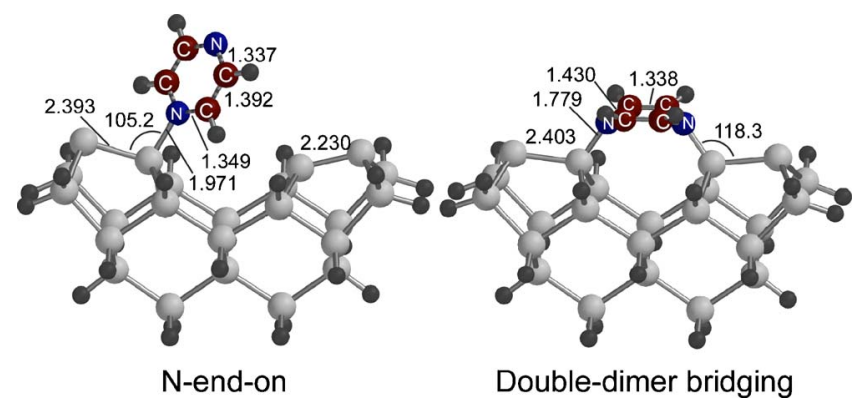

FIG. 4. (Color online) Local minima (i.e., the NEO and DDB configurations) for the pyrazine $/ \mathrm{Si}_{23} \mathrm{H}_{24}$ model system predicted at the B3LYP/6-31G(d) level of theory. Key bond lengths (in $\AA$ ) and angles (in deg.) are indicated in the models. the pyrazine species. On the other hand, in the NEO model, an $\mathrm{N}$ atom is datively bonded to $\mathrm{Si}$ and a lone pair remains on the pyrazine-free $\mathrm{Si}$ atom in the dimer. Moreover, unpaired electrons are not created in the 1,4-di- $\sigma$ and 2,5-di- $\sigma$ models. Due to the existence of these unpaired electrons, i.e., dangling bonds, in the DDB model, this model is found to be somewhat less stable in the calculation. If the number of unpaired electrons were decreased, the stability of the DDB model would increase. This can be achieved by arranging the DDB configurations continuously along the dimer bond direction.

This lining up of the chemisorption species is indeed found in the STM image shown in Fig. 2(a). The atomicscale STM image in which the protrusions linearly align along the dimer bond direction one-by-one is also shown in Fig. 2(c). The stability of the DDB structure would increase after the molecular line is formed, since the structure has unpaired electrons only at the distal part of the line. In other words, the distal part of the line is highly reactive to subsequent adsorption of pyrazine. Thus, this reaction can be considered as quasi-polymerization with the formation of $1 \mathrm{D}$ molecular chains on a solid surface. A structure similar to the DDB configuration with unsaturated electrons has been reported as a possible configuration for acrylonitrile on $\mathrm{Si}(001)-2 \times 1 ;^{22}$ however, the formation of a line structure is not reported. To the best of our knowledge, this is the first observation on the formation of a self-assembled 1D molecular chain on a semiconductor.

At present, the length of the 1D molecular chain is less than $10 \mathrm{~nm}$. However, the phenomenon of the lining up of pyrazine indicates that an adsorbed pyrazine migrates from a meta-stable configuration to the edge of the self-organizing line. The existence of meta-stable sites, such as the NEO and di- $\sigma$ configurations, would allow the migration of pyrazine at RT by varying the number of electrons that can be provided to a dimer; however, additional theoretical and experimental researches are required for clarifying the migration mechanism. If the velocity of the reaction rate is appropriately controlled by controlling various factors such as the substrate temperature and exposed pressure, the length of the line is elongated.

The DDB structure and 1D molecular chain can be used in many applications. If the $\mathrm{Si}$ surface is used as a reaction field, the provision of $\mathrm{C}=\mathrm{C}$ double bonds on the $1 \mathrm{D}$ molecular chain would assist the further reactions. Namely if additional functional molecules that play an important role in biochemistry or Si based nanotechnology are bonded to the double bonds, 1D chains of the functional device may be fabricated. For such device applications, it would be advantageous that both pyrazine and silicon are not chemical toxic materials.

In summary, we have investigated the chemisorption structure of pyrazine on the $\mathrm{Si}(001)-2 \times 1$ surface at RT. From the STM results, we found that the elliptical protrusions corresponding to the pyrazine species were located at the halfway between the dimer rows along the line connecting the dimer bonds. The pyrazine species are further found in STM image to arrange continuously along the dimer bond 
direction. Multiple scattering analyses of the measured $\mathrm{N} 1 s$ and $\mathrm{C} 1 s$ PED patterns support the DDB model. DFT cluster calculation showed, however, that the NEO model is more stable than the DDB model for a single molecule adsorption. The stability of linearly arranged DDB pyrazine species as found by STM and PED is discussed on the basis of "polymerization" of the adsorbates through dangling bonds at the distal points of the 1D molecular chain.

\section{ACKNOWLEDGMENTS}

The authors gratefully acknowledge K. Mase (IMSS) for supporting our experiments at the beamline. Part of this work is supported by the Grant-in-Aid for Young Scientists (No. 14750020) of Ministry of Education, Culture, Sports, Science and Technology. Experiments at KEK-PF were performed under PF PAC No. 2004G024.
*Corresponding author.

Electronic address: romshimo@rie.shizuoka.ac.jp

${ }^{1}$ D. J. Chadi, Phys. Rev. Lett. 43, 43 (1979).

${ }^{2}$ J. C. Fernandez, W. S. Ying, H. D. Shih, F. Jona, D. W. Jepsen, and P. M. Marcus, J. Phys. C 14, L55 (1981).

${ }^{3}$ P. C. Weakliem, G. W. Smith, and E. A. Carter, Surf. Sci. 232, L219 (1990).

${ }^{4}$ M. A. Filler and S. F. Bent, Prog. Surf. Sci. 73, 1 (2003).

${ }^{5}$ Y. Taguchi, M. Fujisawa, T. Takaoka, T. Okada, and M. Nishijima, J. Chem. Phys. 95, 6870 (1991).

${ }^{6}$ M. J. Kong, A. V. Teplyakov, J. Lyubovitsky, and S. F. Bent, Surf. Sci. 411, 286 (1998).

${ }^{7}$ U. Birkenheuer, U. Gutdeutsch, and N. Rösch, Surf. Sci. 409, 213 (1998).

${ }^{8}$ M. Shimomura, M. Munakata, K. Honma, S. M. Widstrand, L. Johansson, T. Abukawa, and S. Kono, Surf. Rev. Lett. 10, 499 (2003).

${ }^{9}$ G. P. Lopinski, D. J. Moffatt, and R. A. Wolkow, Chem. Phys. Lett. 282, 305 (1998).

${ }^{10}$ P. L. Silvestrelli, F. Ancilotto, and F. Toigo, Phys. Rev. B 62, 1596 (2000).
${ }^{11}$ W. A. Hofer, A. J. Fisher, G. P. Lopinski, and R. A. Wolkow, Phys. Rev. B 63, 085314 (2001).

${ }^{12}$ X. Lu, J. Wu, N. Wang, and Q. Zhang, New J. Chem. 26, 160 (2002).

${ }^{13}$ Q. Li and K. T. Leung, Surf. Sci. 541, 113 (2003).

${ }^{14}$ H. G. Huang, J. Y. Huang, Y. S. Ning, and G. Q. Xu, J. Chem. Phys. 121, 4820 (2004).

${ }^{15}$ Y. Chen and M. A. Van Hove, http://electron.lbl.gov/mscdpack/.

${ }^{16}$ GAUSSIAN 03, Revision C.02, M. J. Frisch et al., Gaussian, Inc., Wallingford CT, 2004.

${ }^{17}$ A. D. Becke, J. Chem. Phys. 98, 5648 (1993).

${ }^{18}$ C. Lee, W. Yang, and R. G. Parr, Phys. Rev. B 37, 785 (1988).

${ }^{19}$ P. C. Hariharan and J. A. Pople, Chem. Phys. Lett. 66, 217 (1972).

${ }^{20}$ M. M. Francl, W. J. Pietro, W. J. Hehre, J. S. Binkley, M. S. Gordon, D. J. DeFrees, and J. A. Pople, Chem. Phys. 77, 3654 (1982).

${ }^{21}$ R. J. Hamers, R. M. Tromp, and J. E. Demuth, Phys. Rev. B 34, 5343 (1986).

${ }^{22}$ M. P. Schwartz and R. J. Hamers, Surf. Sci. 515, 75 (2002). 